\title{
Partikelbruchvorhersage an einem dynamischen Übergabesystem und Vergleich mit einer herkömmlichen Schurre mittels DEM
}

\author{
Michael Denzel und Michael Prenner
}

Lehrstuhl für Bergbaukunde, Bergtechnik und Bergwirtschaft - Fördertechnik und Konstruktionslehre, Montanuniversität Leoben, Leoben, Österreich

Eingegangen 11. Januar 2022; angenommen 12. Januar 2022; online publiziert 25. Januar 2022

Zusammenfassung: Zur Reduktion des Partikelbruchs wird eine dynamische Übergabevorrichtung „FlowScrape“ vorgestellt, die aus mehreren zu einer Muldung zusammengeführten gleichlaufenden Gummiketten besteht und mittels Reibrad am abführenden Gurt angetrieben wird. Um den partikelschonenden Effekt zu quantifizieren, wurden vergleichende Versuche mit einer herkömmlichen Übergabeschurre als Referenz durchgeführt. Die Versuche wurden je nach Größenfraktion jeweils mit $10 \mathrm{~kg}$ bzw. $5 \mathrm{~kg}$ Hochofensinter durchgeführt und zeigen eine deutliche Reduktion des Partikelbruchs. Vor allem die Feinmaterialerzeugung konnte reduziert werden. Die Feinmaterialerzeugung bei Übergabe mit dem „FlowScrape“ reduziert sich um annähernd $50 \%$ gegenüber der herkömmlichen Übergabeschurre. Zusätzlich wurden beide Übergabesysteme mithilfe der DEM simuliert. Ein Modell zur Vorhersage des Partikelbruchs wurde vorgestellt, welches auf den Spitzenwerten der Kontaktkraft jedes einzelnen Partikels basiert und mithilfe von Bruchversuchen kalibriert wurde. Die Bruchversuche wurden mit einem speziell entwickelten automatischen Prüfstand zur Einzelpartikelbruchprüfung durchgeführt, welcher eine effiziente Analyse der Bruchcharakteristik von Schüttgütern ermöglicht. Die Ergebnisse der Partikelbruchvorhersage mittels Simulation weichen bei den kleineren Größenfraktionen inklusive Feinmaterial nur geringfügig von den Versuchsergebnissen ab. Bei den größeren Fraktionen kommt es aber teilweise zu signifikanten Abweichungen von den Versuchsergebnissen. Deshalb wird derzeit mithilfe eines anderen Ansatzes ein neues Partikelbruchmodell für die DEM entwickelt, welches es ermöglicht

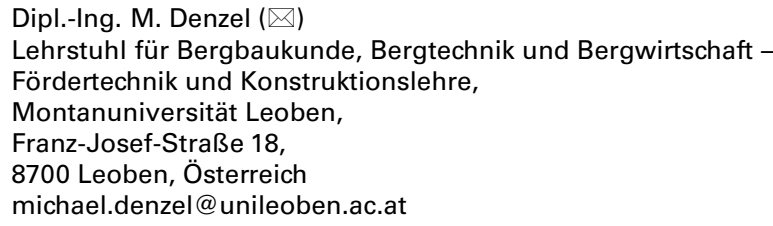

den Partikelbruch selbst zu simulieren und somit eine höhere Genauigkeit erzielt werden kann.

Schlüsselwörter: Übergabeschurre, Partikelbruch, Bruchmodell, Diskrete Elemente Methode, Hochofensinter, Rückgut

Particle Breakage Prediction on a Dynamic Transfer System and Comparison with a Conventional Chute Using DEM

Abstract: To reduce particle breakage, the dynamic transfer system "FlowScrape" is presented, which consists of several co-rotating rubber chains brought together to form a trough and is driven by a friction wheel on the discharging belt. In order to quantify the particle preserving effect, comparative tests were done using a conventional transfer chute as a reference. The tests were carried out with $10 \mathrm{~kg}$ or $5 \mathrm{~kg}$ blast furnace sinter, depending on the size fraction. The results show a significant reduction in particle breakage. Especially the production of fines could be reduced. The fine material generation during transfer with the "FlowScrape" is reduced by nearly $50 \%$ compared to the conventional transfer chute. In addition, both transfer systems were simulated using the DEM. A particle breakage prediction model was presented based on the peak contact force of each particle and was calibrated by breakage tests. The breakage tests were performed using a specially designed automated single particle impact tester, which allows an efficient analysis of the breakage behavior of bulk materials. The results of the particle breakage prediction by simulation only slightly differ from the test results for the smaller size fractions including fines. For the larger fractions, however, there are sometimes significant deviations from the experimental results. Therefore, a new particle breakage model for the DEM is currently being developed using a different approach, which makes it possible to sim- 


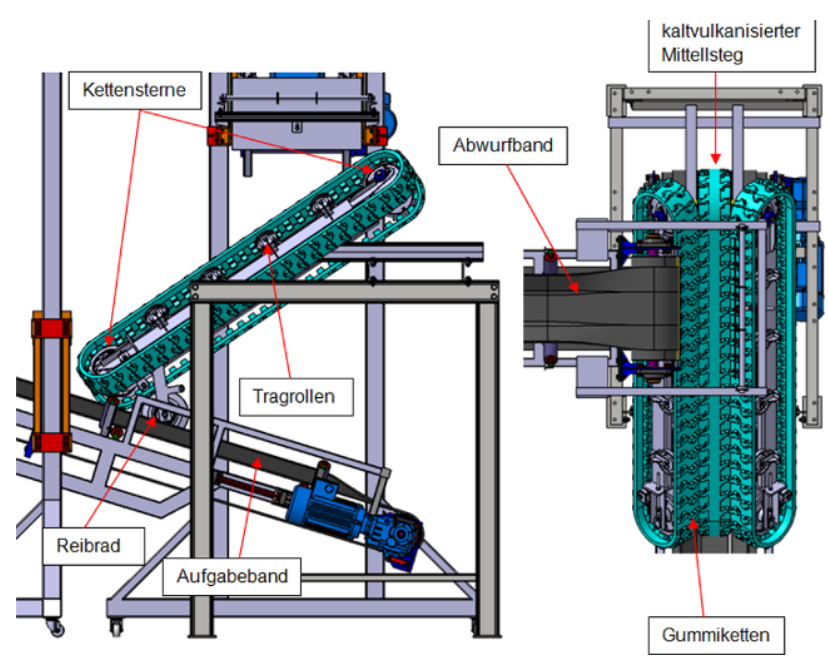

Abb. 1: Erster Prototyp der dynamischen Übergabeeinrichtung „FlowScrape“ [4]

ulate particle breakage itself and thus achieve a higher accuracy.

Keywords: Transfer chute, Particle breakage, Breakage model, Discrete Element Method, Blast furnace sinter, Return fines

\section{Einleitung}

Hochofensinter weist ein sehr inhomogenes Materialverhalten auf und ist je nach Herstellungskonfiguration mehr oder weniger fest bzw. fragil. Diese Eigenschaft führt innerhalb der notwendigen Transport-, Lager- und Siebprozesse zu Partikelbrüchen. Im Nachfolgenden wird speziell auf das Bruchverhalten an Übergabestellen zwischen Gurtbandförderern eingegangen. An Übergabestellen besitzt das Schüttgut aufgrund des Höhenunterschiedes und der Fördergeschwindigkeit der beteiligten Gurtbandförderer oft

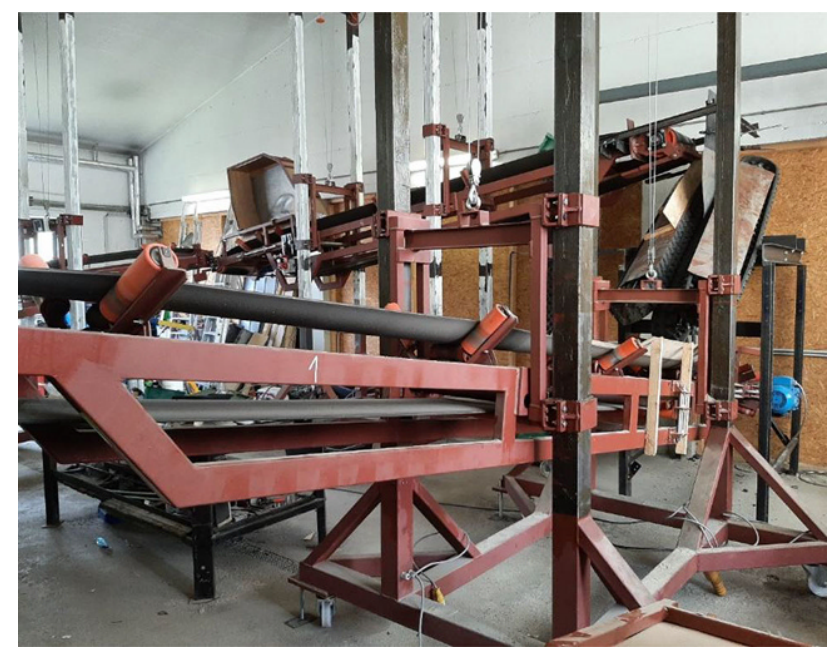

Abb. 2: Lehrstuhleigener Förderkreislauf mit „FlowScrape“ am Prüfstand

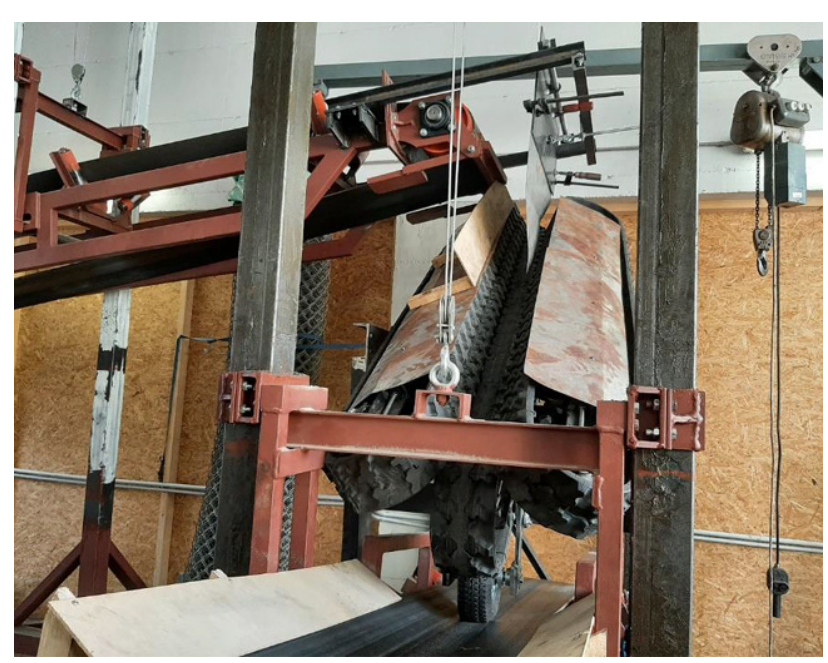

Abb. 3: „FlowScrape" als Übergabeeinrichtung

einen hohen Energieinhalt. Durch den Übergabevorgang kann das Schüttgut stark abgebremst werden, wodurch die Bewegungsenergie zum Großteil in Schüttgut- und Anlagenverschleiß umgewandelt wird. Im Zuge des von der EU geförderten RFCS-Projektes MinSiDeg wird derzeit nach Lösungen gesucht, den Sinterzerfall signifikant zu reduzieren. Um das Bruchverhalten des Sinters besser zu verstehen, wurde eine "Schüttgutkanone" entwickelt, die einzelne Sinterpartikel geschwindigkeitsgesteuert gegen ein definiertes Hindernis schießt und den dabei entstehenden Kornzerfall misst. Die Messergebnisse dienen auch zur Bruchparameterkalibration, der für das Projekt notwendigen durchzuführenden "Diskrete Elemente Simulationen“. Der Lehrstuhl entwickelt derzeit in einem Parallelprojekt in Kooperation mit den Firmen ScrapeTec Trading GmbH,



Abb. 4: Herkömmliche Übergabeschurre 

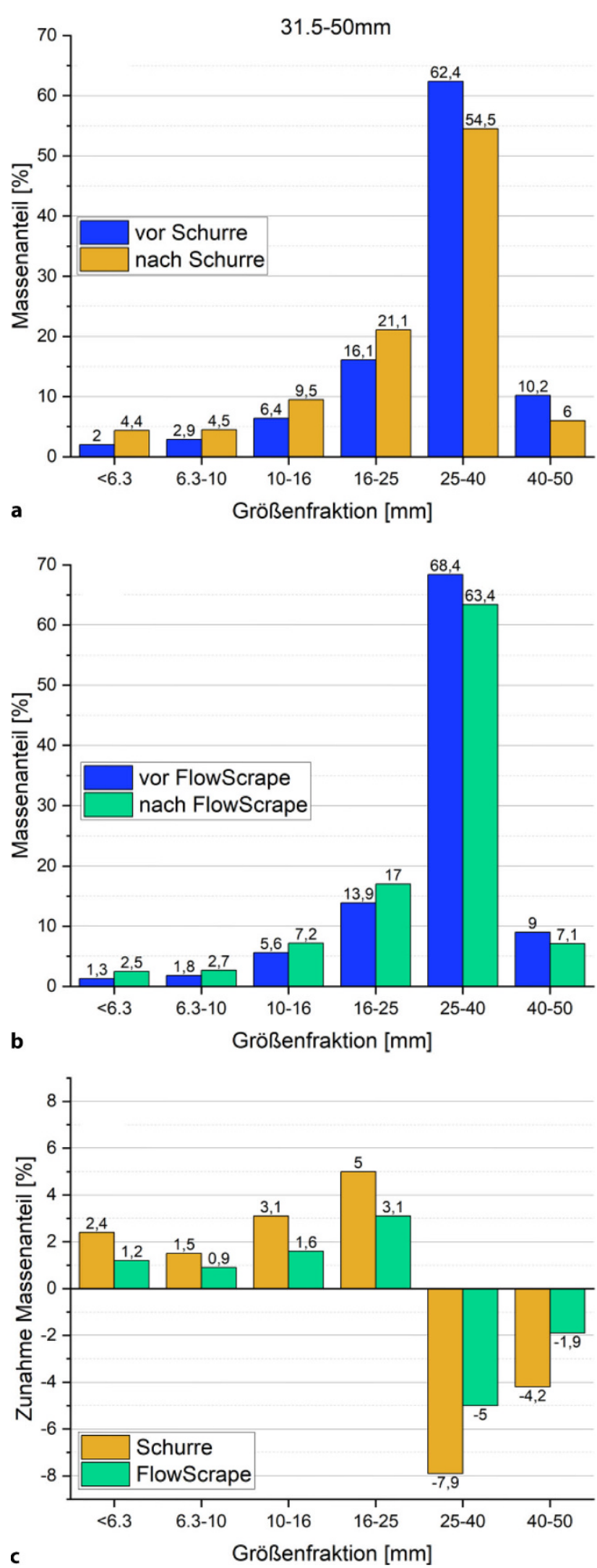

Abb. 5: Partikelgrößenverteilung Größenfraktion 31,5-50 mm a vor und nach Schurre b vor und nach „FlowScrape“ $\mathbf{c}$ Änderungen bei Übergabe mit Schurre und "FlowScrape"
ScrapeTec GmbH und Wanggo Gummitechnik GmbH eine dynamische Übergabeeinrichtung, die in Bezug auf die Anwendbarkeit zur Partikelbruchreduktion hin untersucht wurde.

Der folgende Beitrag ist eine überarbeitete Version von [1], wobei neue Erkenntnisse und weitere Details mit eingearbeitet wurden. Neu gegenüber [1] ist vor allem die zweite Versuchsreihe mit der nächst kleineren Größenfraktion 16-31,5 mm und die weiterführenden Simulationen mit dem Ziel die Genauigkeit der Partikelbruchvorhersage zu erhöhen. Außerdem werden weitere Details bezüglich des noch in Entwicklung befindlichen neuen Partikelbruchmodells für die Diskrete Elemente Methode (DEM) beschrieben.

\section{Dynamisches Übergabesystem}

Das Funktionsprinzip und der Aufbau des Systems sind in Abb. 1 dargestellt. Die dynamische Übergabeschurre "FlowScrape" besteht im Wesentlichen aus einer Art Gurtförderer, der zwischen dem Abwurfband und dem Aufgabeband montiert wird. Das Übergabesystem wird aus mindestens drei zu einer Muldung zusammengeführten Gummiketten aufgebaut. Der Kettenaufbau und die dazugehörigen Tragorgane sind an die Funktion von Gummikettenbaggerfahrwerken angelehnt. Eine kardanische Verbindung der Kettenräderachsen (Turasse) sorgt für den Gleichlauf der Ketten. Der Antrieb der Übergabeketten kann auf zwei Arten realisiert werden. Grundsätzlich sollte der Antrieb über ein Reibrad durch das Aufgabeband passieren. Die mittlere Kette wird dafür auf das Reibrad gepresst, wobei das Reibrad zusätzlich auf das Aufgabeband gepresst wird. Das Kettensystem ist dafür drehbar (parallel zu den Trommelachsen des Aufgabebandes) aufgehängt, und sollte die Eigenmasse der Übergabeketten nicht ausreichen, kann zusätzlich eine Anpressvorrichtung implementiert werden. Alternativ zum Reibradantrieb kann das System auch elektromotorisch angetrieben werden. Je nach Schüttguteigenschaften des transportierten Schüttgutes kann die Abdichtung der Kettenlängsübergänge wie folgt realisiert werden. Bei Schüttgütern ohne Feinanteil können die Kettenlängskanten zur Abdichtung aneinandergepresst werden. Aufgrund von Fertigungstoleranzen der Ketten entstehen beim Aneinanderpressen der Kanten aber immer kleine sich öffnende und wieder schließende Spalten, durch die Teile der Feinfraktion durchrieseln können. Bei feinkörnigem Schüttgut werden die äußeren Ketten mit der mittleren Kette überlappt ausgeführt, um eine Abdichtung zu gewährleisten und somit ein Durchrieseln des feinkörnigen Schüttgutes zu vermeiden. Je nach Größe des erforderlichen Neigungswinkels der Übergabeketten können diese mit oder ohne Querstollen ausgeführt werden. Die dynamische Übergabevorrichtung wurde zum Patent angemeldet (siehe [2]). Eine detaillierte Beschreibung des Systems und Erkenntnisse aus der Prototypenentwicklung und den ersten Versuchsreihen sind in [3] und [4] beschrieben.

Das Übergabesystem hat neben der Reduktion des Partikelbruchs noch weitere Vorteile. Im Vergleich zu herkömmli- 

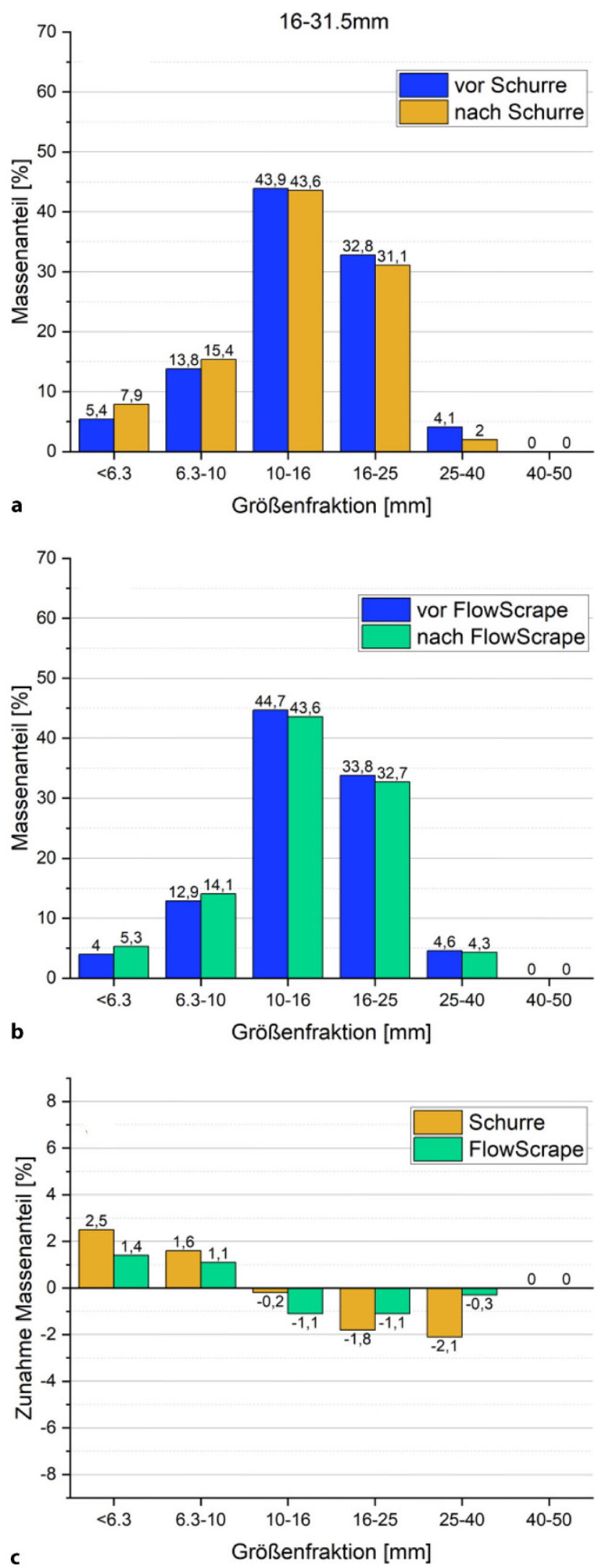

Abb. 6: Partikelgrößenverteilung Größenfraktion 16-31,5 mm a vor und nach Schurre b vor und nach "FlowScrape" $\mathbf{c}$ Änderungen bei Übergabe mit Schurre und „FlowScrape“ chen starren Übergabeschurren ist der Schurrenverschleiß nicht "punktuell,, sondern verteilt sich auf die gesamte umlaufende Kettenoberfläche. Eine signifikant längere Standzeit ist dadurch zu erwarten. Bei ausreichendem Massenstrom und Höhenunterschied reichen die entstehenden Hangabtriebskräfte aus, um die Bewegungswiderstände des Kettensystems zu überwinden. Die dynamische Schurre muss dann durch das Aufgabeband oder den elektrischen Antrieb gebremst werden. In diesem Fall tritt eine gewisse Energierückgewinnung auf [3]. In Kombination mit der Reduktion der Antriebsleistung des Aufgabebands und der Energierückgewinnung entsteht somit ein energieeffizientes System. Aufgrund der konstruktiven Ausführung sind Verstopfungen durch Anbackungen unwahrscheinlich. Lediglich massive unbeabsichtigte Überschüttungen können zu Problemen führen. Im Vergleich zu herkömmlichen Beschleunigungsbändern verfügt das Kettensystem über eine zentrale Führungseinrichtung an der Kettenlaufseite. Durch diese Zwangsführung kann es im Gegensatz zu Beschleunigungsbändern zu keinem Gurtschieflauf unabhängig von der Aufgaberichtung kommen.

Das System ist immer mit einer gerichteten Übergabe in Förderrichtung des Aufgabebands verbunden (SoftLoading). Dadurch muss das Schüttgut weniger beschleunigt werden (Reduktion der Antriebsleistung), und es tritt eine geringere Gurtbelastung und somit geringerer Verschleiß des Aufgabeförderers auf. Im Gegensatz zu einfachen Rutschen kann dieser Soft-Loading-Effekt mit dem "FlowScrape" auch bei geringen Fallhöhen erzielt werden. Rutschen würden bei geringen Fallhöhen aufgrund der großen Geschwindigkeitsabnahme des Schüttguts zum Verstopfen neigen.

\section{Vergleichende Versuche mit herkömmli- cher Übergabeschurre}

Um die Reduktion der Belastungen auf das Schüttgut während der Übergabe mit dem „FlowScrape“ zu quantifizieren, wurde der daraus resultierende Partikelbruch mit jenem bei Übergabe mit einer herkömmlichen Schurre, welche in dieser Form auch zum Transport von Hochofensinter Anwendung finden, verglichen. Die herkömmliche Übergabeschurre besteht aus einer quaderförmigen Holzkonstruktion mit einer Prallplatte aus Stahl an der Hinterwand und zusätzlichen Abdeckungen in Gurtnähe, um Materialverlust zu verhindern (siehe Abb. 4). Der Großteil des Schüttgutes kollidiert dabei mit der Prallpatte und fällt anschließend auf den abführenden Gurt. Die Gesamtfallhöhe beträgt $1600 \mathrm{~mm}$. Solche Fallschächte werden alternativ zu "Rockboxen" verbaut. Bei einer "Rockbox" wird eine bewusste Materialanhäufung herbeigeführt, wodurch durch Aufprall in das somit erzeugte Schüttbett der Verschleiß der Schurre reduziert wird, dafür aber aufs Schüttgut selbst übertragen wird und die Degradation dessen teilweise fördert. $\mathrm{Da}$ "Rockboxen" allerdings zum Verstopfen neigen und somit Probleme verursachen, werden bestehende "Rockboxen" in einigen Fällen durch herkömmliche Schurren bzw. Fallschächte ersetzt. 


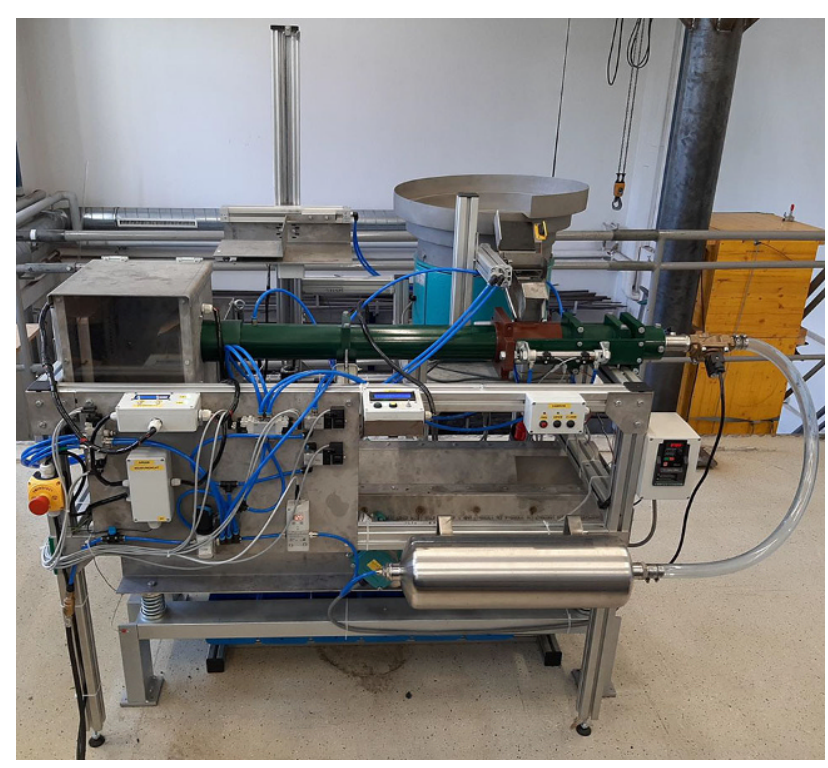

Abb. 7: Automatischer Prüfstand zur Einzelpartikelbruchprüfung, näher beschrieben in [6]

Die Versuchsreihen wurde am lehrstuhleigenen Förderkreislauf durchgeführt (siehe Abb. 2). Dieser besteht aus vier Gurtbandförderern mit $400 \mathrm{~mm}$ Breite, dessen Neigungen, Muldungen und Geschwindigkeiten jeweils individuell verstellbar sind. Drei der vier Übergabestellen sind mit einfachen Prallplatten ausgestattet. Eine Übergabestelle ist als Prüfstand vorgesehen, um dort verschiedenste Übergabesysteme montieren und im Kreislauf testen zu können.

Es wurden zwei Versuchsreihen mit den vom Hersteller vorgesiebten Größenfraktionen $16-31,5 \mathrm{~mm}$ und 31,5-50 mm durchgeführt, wobei letztere die kritische Fraktion für den Partikelbruch darstellt. Es wurden pro Versuch jeweils $10 \mathrm{~kg}(31,5-50 \mathrm{~mm})$ bzw. $5 \mathrm{~kg}(16-31,5 \mathrm{~mm})$ Material verwendet. Die tatsächliche Partikelgrößenverteilung innerhalb dieser vorgesiebten Größenfraktionen wurde jeweils vor den Versuchen mittels einer eigens konzipierten partikelschonenden Sortierrinne ermittelt, welche in [5] und [6] näher beschrieben ist. Da die Partikelgrößenverteilung innerhalb der vom Hersteller bereitgestellten Größenfraktion schwankt und die Klassierung mittels Sortierrinne von der Ausrichtung der Partikel in der Sortierrinne abhängt, wurden die Proben jeweils dreimal sortiert und davon das arithmetische Mittel als Partikelgrößenverteilung vor dem Bruch definiert. Bei gleichbleibenden Gurtgeschwindigkeiten und Fallhöhen wurden jeweils sechs Versuche mit dem "FlowScrape" (Abb. 3) und jeweils fünf Versuche mit einer herkömmlichen Schurre (Abb. 4) als Übergabeeinrichtung durchgeführt. Beim "FlowScrape" wurde zusätzlich eine Prallplatte am oberen Ende montiert, um Materialverlust zu verhindern und den "FlowScrape“ mittig zu beladen. Die Schüttgutprobe wurde jeweils am Anfang des zuführenden Gurtes händisch aufgegeben und verteilt, sodass keine Partikel übereinanderliegen. Die Gurtgeschwindigkeiten des zu- und abführenden Gurtes betrugen 1,5m/s. Die Schüttgutprobe wurde am Ende des abführenden Gurtes in einer Kunststoffwanne gesammelt. Die Fallhöhe in die Kunststoffwanne war ebenfalls bei allen Versuchen gleich und betrug in etwa $0,5 \mathrm{~m}$. Im Anschluss wurde wiederum die Partikelgrößenverteilung nach dem Versuch durch dreimaliges Sortieren in der Sortierrinne gemessen.

In Abb. 5a ist die massenbezogenen Partikelgrößenverteilungen der Versuchsreihe mit der Größenfraktion $31,5-50 \mathrm{~mm}$ vor und nach der Übergabe mit einer herkömmlichen Schurre dargestellt. Die Partikelgrößenverteilung vor und nach Übergabe mit dem "FlowScrape" ist in Abb. 5b ersichtlich. Die Werte ergeben sich jeweils aus dem arithmetischen Mittel aller sechs bzw. fünf Versuche. Einen direkten Vergleich des Partikelbruchs zwischen "FlowScrape" und herkömmlicher Übergabeschurre zeigt Abb. 5c. Hierbei ist die jeweilige Zunahme der Massenanteile pro Größenfraktion abgebildet. Die Ergebnisse zeigen, dass sowohl bei "FlowScrape" als auch bei der herkömmlichen Übergabeschurre die größeren Partikel eher zum Bruch neigen und die Massenanteile bei den Größenfraktionen $25-40 \mathrm{~mm}$ und $40-50 \mathrm{~mm}$ jeweils abnehmen. Zugleich nehmen die Massenanteile der kleineren Größenfraktionen $<25 \mathrm{~mm}$ zu.

Der signifikante Unterschied bei den Zu- und Abnahmen der Massenanteile zwischen "FlowScrape“ und herkömmlicher Übergabeschurre beweist, dass bei Übergabe mit dem "FlowScrape" weniger Partikelbruch stattfindet. Vor allem beim für die Stahlindustrie so relevanten Feinmaterial (Rückgut, $<6,3 \mathrm{~mm}$ ) ist der Unterschied gravierend. Demnach wird bei der Versuchsreihe mit der Größenfraktion 31,5-50 mm bei Übergabe mit dem „FlowScrape" $50 \%$ weniger Feinmaterial erzeugt im Vergleich zur Übergabe mit einer herkömmlichen Schurre.

In Abb. 6 sind, wie bereits für Abb. 5. beschrieben, die Versuchsergebnisse für die Versuchsreihe mit der Größenfraktion $16-31,5 \mathrm{~mm}$ dargestellt. Hierbei ist deutlich weniger Partikelbruch zu verzeichnen als bei der Versuchsreihe mit der Größenfraktion 31,5-50 mm. Dies stimmt mit den Erwartungen überein, da größere Partikel generell eher zum Bruch neigen als kleinere und auch in der Industrie die größere Fraktion 31,5-50 mm als kritischer angesehen wird. Bemerkenswert ist allerdings, dass auch in dieser Versuchsreihe sowohl bei der herkömmlichen Übergabeschurre als auch beim „FlowScrape“ ähnlich viel Rückgut entsteht wie bei der Versuchsreihe mit der Größenfraktion 31,5-50 mm. Hierbei wird vermutet, dass dies nicht nur durch Volumenbruch der größeren Partikel zustande kommt, sondern auch der Partikelabrieb, vor allem bei kleineren Partikeln, einen relevanten Einfluss auf die Rückgutproduktion hat. Da bei der Versuchsreihe mit der Größenfraktion 16-31,5 mm die Partikelanzahl deutlich höher als bei der Größenfraktion 31,5-50 mm ist, steigt somit auch der Einfluss des Partikelabriebs durch Partikelinteraktionen.

\section{Bruchversuche}

Zur Analyse des Bruchverhaltens und für die Kalibration des Simulationsmodells wurden Einzelpartikelbruchversuche mit dem in den Versuchen verwendeten Hochofensinter durchgeführt. Dabei wurde das Bruchverhalten von jeweils 300 Partikeln der Größenfraktionen 31,5-50 mm und $16-31,5 \mathrm{~mm}$ mittels eines speziell entwickelten auto- 

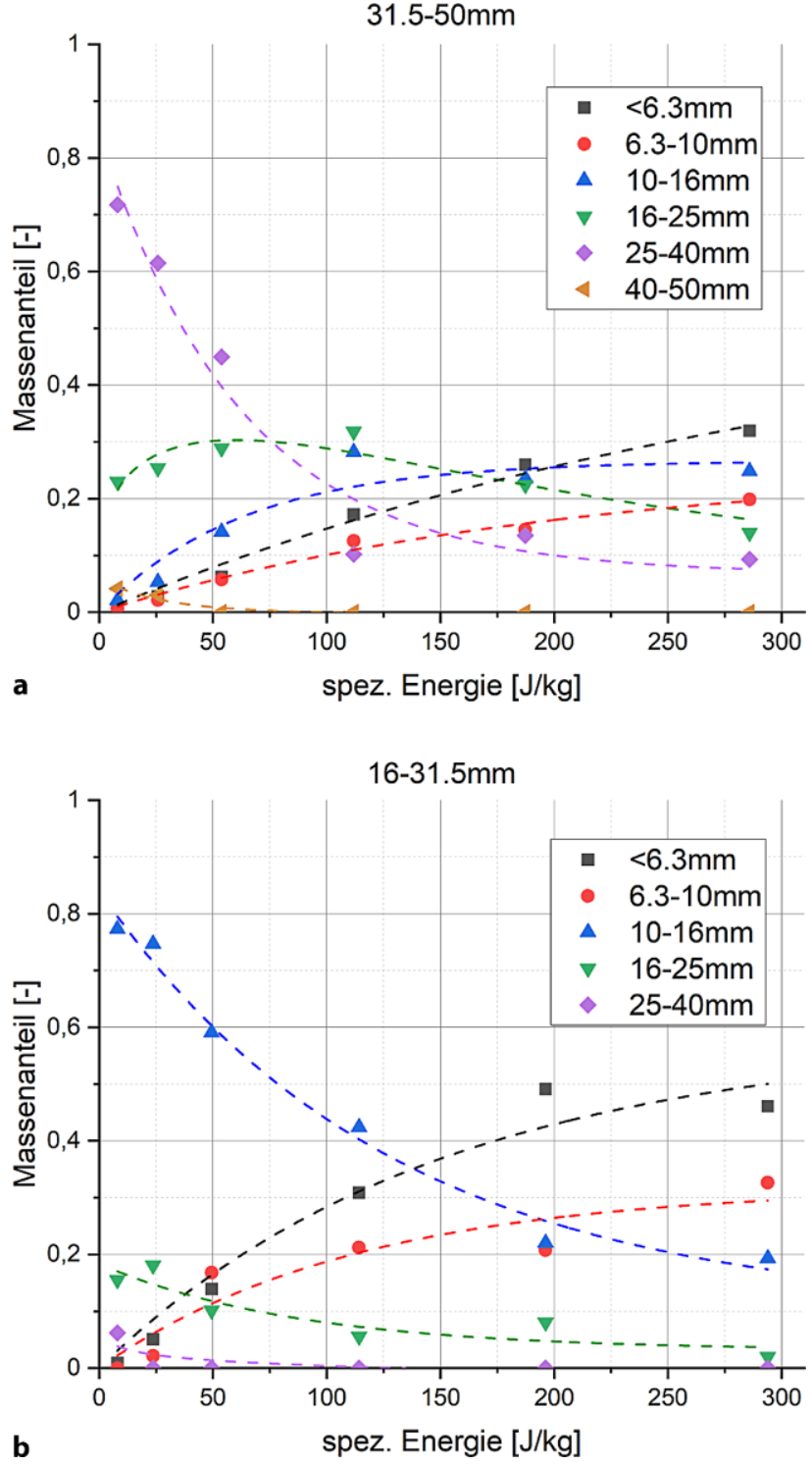

Abb. 8: Bruchcharakteristik von Hochofensinter a 31,5-50 mm b $16-31,5 \mathrm{~mm}$

matischen Einzelpartikelbruchprüfstandes analysiert [6]. Der Prüfstand (Abb. 7) besteht aus einer Vereinzelung mittels Schwingtopf, Wiegestation, Beladungsmechanismus, Druckluftkanone und einer inkludierten Fragmentanalyse. Alle Module sind vollautomatisiert, womit eine zeiteffiziente Versuchsdurchführung (Versuchsdauer 50-70s) ermöglicht wird. Die Partikel werden dabei einzeln verwogen und mittels Druckluftkanone mit einer definierten Geschwindigkeit gegen eine Stahlplatte geschossen. Dabei wird die tatsächliche Aufprallgeschwindigkeit mittels Lichtschranken gemessen und die Fragmente schonend in die eigens dafür entwickelte Sortierrinne geleitet, um die Partikelgrößenverteilung nach dem Bruch zu analysieren. Der Prüfstand ist in [5] und [6] näher beschrieben. Die Ergebnisse der Bruchversuche sind in Abb. 8 ersichtlich. Mit zunehmendem massenspezifischen Energieeintrag nimmt der Massenanteil der größeren Körner exponentiell ab und der Anteil der kleineren Fraktionen nimmt dementspre- chend zu. Eine detailliertere Interpretation der Ergebnisse und weitere Zusammenhänge zur Charakterisierung des Bruchverhaltens von Hochofensinter sind ebenfalls in [6] beschrieben.

\section{Simulation}

Sowohl die Übergabe mittels „FlowScrape“ (Abb. 9) als auch mittels herkömmlicher Schurre (Abb. 10) wurden mithilfe der DE-Software EDEM simuliert. Die Stollenbewegungen an den Gummiketten des „FlowScrapes" wurden mittels Translation und Rotation entlang der Umlaufkurve realisiert. Die verwendeten Größenfraktionen 31,5-50 mm und $16-31,5 \mathrm{~mm}$ wurde durch runde Partikel mit gemittelten Durchmessern von $40 \mathrm{~mm}$ bzw. $22 \mathrm{~mm}$ dargestellt. Aus den Bruchversuchen sind die durchschnittlichen Partikelmassen dieser Größenfraktionen bekannt (59,9g bzw. 10,2 g). Die daraus resultierende Partikelzahl (167 bzw. 490) bei einer Schüttgutprobe von $10 \mathrm{~kg}$ bzw. $5 \mathrm{~kg}$ wurde auch in der Simulation auf den zuführenden Gurtbandförderer platziert. Die Dichte $\left(1788 \mathrm{~kg} / \mathrm{m}^{3}\right.$ bzw. $\left.1830 \mathrm{~kg} / \mathrm{m}^{3}\right)$ wurde an Partikelanzahl und Probenmasse angepasst. Reibungsparameter wurden mittels Schüttkegel- und Abgleitversuchen ermittelt [7]. Die Stoßzahlen für Sinter-Sinter, Sinter- „FlowScrape", Sinter-Gurt und Sinter-Stahl wurden mittels Rücksprungversuchen und Hochgeschwindigkeitsaufnahmen aus zwei Perspektiven bestimmt [8]. Um die Kontaktkräfte auf die einzelnen Partikel ausreichend exakt detektieren zu können, müssen Zeitschritt und Speicherintervall - abhängig von Partikelgröße, E-Modul und Kontaktgeschwindigkeiten - entsprechend klein sein. Ein zu kleiner Zeitschritt führt allerdings zu hohem Rechenaufwand, ein zu kleines Speicherintervall zu einer Datenflut und ebenfalls erhöhten Rechenzeiten.

In [9] wurden die relativen Abbildefehler bei verschiedenen größeren Zeitschritten und Speicherintervallen im Vergleich zu $10^{-7}$ s für Sinterpartikel von 6 und $40 \mathrm{~mm}$ Durchmesser bei in der Sinterförderung üblichen Geschwindigkeiten ermittelt. Ein Optimum zwischen Genauigkeit, Rechenaufwand und Datenmenge wurde bei diesen Partikelgrößen und den in dieser Simulation auftretenden Aufprallgeschwindigkeiten bei einem Zeitschritt von $10^{-5} \mathrm{~s}$ und einem Speicherintervall von $10^{-4} \mathrm{~s}$ gefunden. Die relativen Abbildefehler im Vergleich zu $10^{-7} \mathrm{~s}$ betragen hierbei $6,616 \%(6 \mathrm{~mm})$ und $0,153 \%(40 \mathrm{~mm})$. Die Simulation mit Zeitschritt $10^{-5} \mathrm{~s}$ und Speicherintervall $10^{-4} \mathrm{~s}$ mit Partikeldurchmessern von $22 \mathrm{~mm}$ bzw. $40 \mathrm{~mm}$ ist somit bei den hierbei auftretenden Geschwindigkeiten für die hier angewandte Methode zur Partikelbruchvorhersage ausreichend genau [9].

\section{Partikelbruchvorhersage}

Um den Partikelbruch mittels Simulation vorhersagen zu können, wurde folgender Ansatz gewählt: Ein Partikel bricht, wenn er eine bestimmte Kontaktkraft erfährt. Die dazu benötigten Kontaktkräfte wurden mittels Kalibration der Bruchversuche ermittelt (siehe Abb. 11). Dazu wurde der 


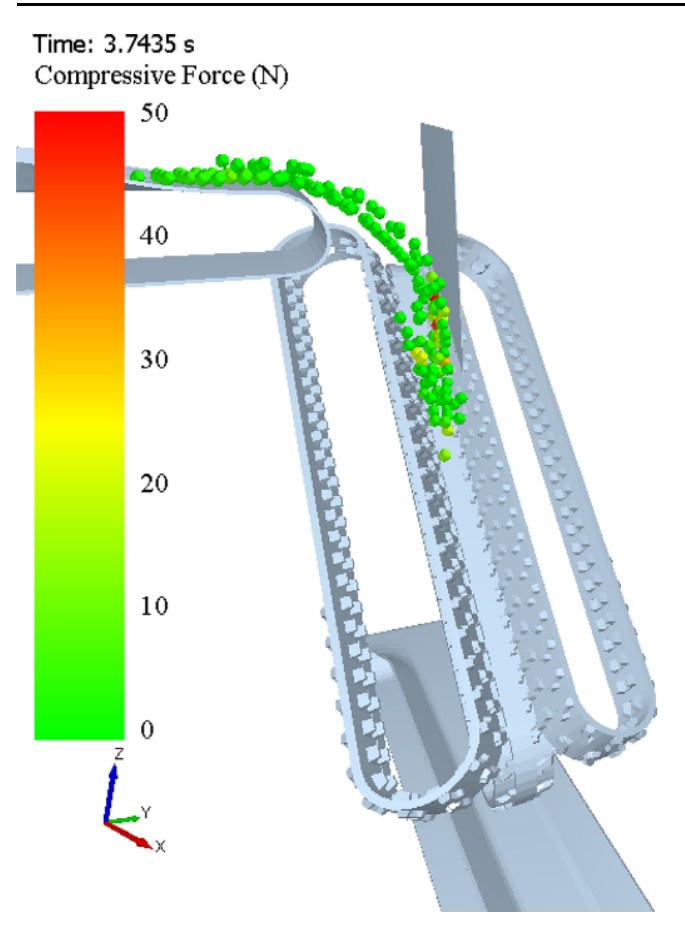

Abb. 9: DE Simulation der Übergabe mittels „FlowScrape“

Prallversuch gegen einen Stahlplatte mit verschiedenen Geschwindigkeiten simuliert (siehe Abb. 11a, b). Zeitschritt und Speicherintervall wurden auch hier mit $10^{-5} \mathrm{~s}$ bzw. $10^{-4} \mathrm{~s}$ definiert, um die Kontaktkraft ausreichend genau zu ermitteln. Mit dem nichtlinearen Zusammenhang zwischen maximaler Kontaktkraft und Geschwindigkeit beim Aufprall (Abb. 11c) und den Ergebnissen der Bruchversuche (Abb. 8) kann anschließend eine Vorhersage über einen eventuellen Partikelbruch getroffen werden. Um einen unnötig niedrigen Zeitschritt und damit höhere Rechenzeiten zu vermeiden, wurden in der Simulation sehr geringe E-Module gewählt. Um das unterschiedliche Dämpfungsverhalten der Kontaktpartner und damit den Einfluss auf die Kontaktkraft zu berücksichtigen, wurden die E-Module von Stahl, Gurt und "FlowScrape" allerdings im richtigen Verhältnis zueinander definiert. Der E-Modul von Sinter wurde dabei auf den kleinstmöglichen Wert gesetzt. Die

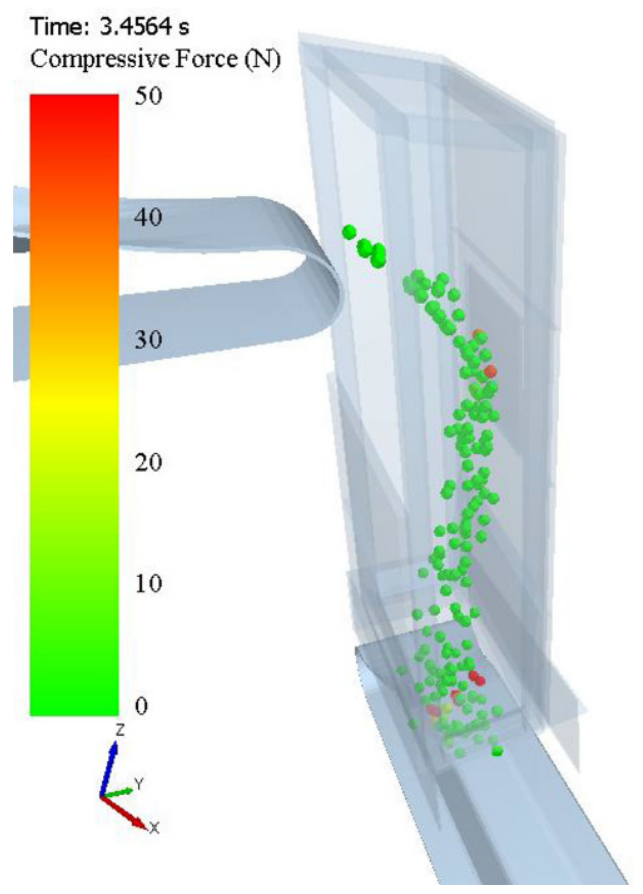

Abb. 10: DE Simulation einer herkömmlichen Übergabeschurre

Stoßzahlen tragen ebenfalls zur Dämpfung bei und wurden anhand der Versuchsergebnisse von [8] definiert.

Für die Partikelbruchvorhersage muss die Kontaktkraft jedes Partikels über die gesamte Förderdauer individuell betrachtet werden. Da EDEM jeweils nur Kontaktkraft oder Partikel-ID exportieren kann, wurde eine Matlab-Routine programmiert, welche die Ergebnistabellen entsprechend verknüpft, sodass für jeden Partikel jederzeit die Kontaktkraft ermittelt werden und der Kontaktkraftverlauf evaluiert werden kann (siehe Abb. 12). Sowohl beim "FlowScrape“ als auch bei der herkömmlichen Übergabeschurre gibt es jeweils zwei Ereignisse die hauptsächlich für den Bruch verantwortlich sind. Die Kollision mit der Prallplatte bzw. die Aufgabe auf den "FlowScrape“ und der Fall auf den abführenden Gurt. Somit wurden jeweils der größte und zweitgrößte Spitzenwert der Kontaktkraft (mit entsprechender Prominenz) für jeden Partikel ermittelt. Anschließend wurden Kraftintervalle definiert und die Anzahl der Partikel mit
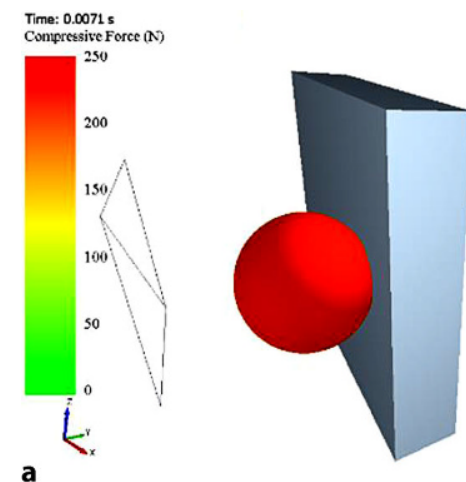

a
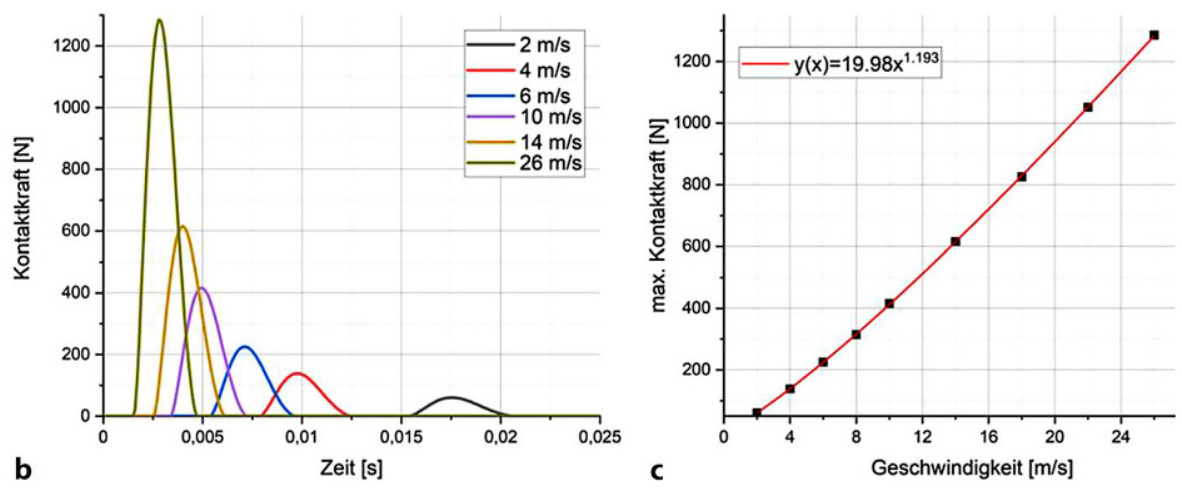

Abb. 11: Kalibration des Bruchversuchs a Simulation b Kontaktkraft bei verschiedenen Geschwindigkeiten c Zusammenhang zwischen max. Kontaktkraft und Geschwindigkeit $(31,5-50 \mathrm{~mm})$ 



Abb. 12: Exemplarischer Kontaktkraftverlauf eines einzelnen Partikels ( $40 \mathrm{~mm}$ für 31,5-50 mm) an herkömmlicher Schurre und "FlowScrape" ab Abwurfzeitpunkt vom Gurtbandförderer

Spitzenwert im jeweiligen Kraftintervall gezählt. Dafür wurde jeweils für den größten (siehe Abb. 13) und zweitgrößten Spitzenwert ein Histogramm erstellt. Wie in Abb. 13 ersichtlich, sind die maximal auftretenden Kontaktkräfte jeweils log-normal-verteilt. Der Mittelwert des Kraftintervalls wurde dann mithilfe des Zusammenhangs in Abb. 11c in eine korrespondierende Geschwindigkeit umgerechnet. Allein mit der Geschwindigkeit kann die massenspezifische Energie $[\mathrm{J} / \mathrm{kg}]$ berechnet werden und anhand der Bruchversuchsergebnisse (Abb. 8) die zu erwartende Partikelgrößenverteilung nach dem Bruch ermittelt werden. Die berechnete Partikelgrößenverteilung nach dem entsprechenden Spitzenwert wurde dann mit der Partikelanzahl pro Kraftintervall gewichtet, womit sich die zu erwartende Partikelgrößenverteilung der gesamten Schüttgutprobe nach der größten auftretenden Belastung ergibt. Der Vorgang wurde für den zweitgrößten Spitzenwert der Kontaktkraft wiederholt und die Zu- bzw. Abnahmen der Massenanteile der jeweiligen Größenfraktionen zu den Ergebnissen des größten Spitzenwertes addiert.

Die Simulationsergebnisse zeigen ebenfalls den partikelschonenden Effekt bei Übergabe mit dem „FlowScrape“. Der Vergleich der Simulationsergebnisse der Größenfraktion $31,5-50 \mathrm{~mm}$ mit den dazugehörigen Versuchen in Abb. 14 zeigt teilweise signifikante Abweichungen bei den größeren Fraktionen, aber eine nur geringe Abweichung bei den kleineren Fraktionen. Vor allem beim Feinmaterial wird im Versuch nur 0,3 bzw. 0,2\% mehr Feinmaterial produziert, als mittels Simulation vorhergesagt. Dies kann daran liegen, dass der Fall in die Kunststoffwanne am Ende des abführenden Gurtes in der Simulation nicht berücksichtig worden ist und beim 3-maligen Sortieren der Fragmente nach dem Versuch zusätzlich Feinmaterial erzeugt wird.

Die Kontaktkräfte in der Simulation mit der Größenfraktion 16-31,5 mm wurden mittels einer eigenen Kalibrationskurve (ähnlich Abb. 11c) ebenfalls in korrespondierende Geschwindigkeiten und massenspezifische Energien umgerechnet. Die prognostizierte Rückguterzeugung liegt auch hier etwas unter dem tatsächlichen Wert aus den Versuchen. Bis auf die Rückgutproduktion konnten bei der Par-

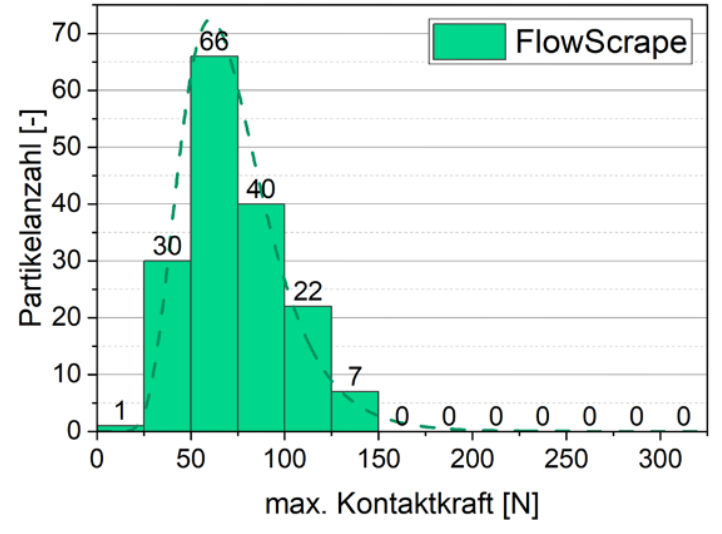

Abb. 13: Belastungsprofile bei herkömmlicher Schurre und „FlowScrape"

tikelbruchvorhersage für die Größenfraktion 16-31,5 mm mit der vorab beschriebenen Methodik allerdings keine zufriedenstellenden Ergebnisse erzielt werden, weshalb diese auch nicht abgebildet sind. Dies liegt vermutlich daran, dass die hierbei auftretenden spezifischen Energieeinträge kaum zu signifikanten Bruch in dieser Größenfraktion führen, da kleinere Partikel generell weniger zum Bruch neigen als große. Es wird festgestellt, dass die hier angewandte Methodik mit den zur Verfügung stehenden Ergebnissen der Bruchversuche für geringe Belastungen zu ungenau ist. Für eine genauere Partikelbruchvorhersage in diesen spezifischen Energiebereichen, sind weitere Bruchversuche im Niedrig-Energiebereich nötig.

\section{Weitere Simulationen}

In [10] wurden weitere Simulationen durchgeführt, um teilweise mit den in [1] vorgeschlagenen Optimierungen die Genauigkeit der Partikelbruchvorhersage zu erhöhen und somit die Abweichungen zu den Realversuchen zu reduzieren. Hierbei wurde sowohl die Übergabe mittels „FlowScrape" als auch mit der herkömmlichen Schurre simuliert. In [1] wird eine Verbesserung durch Anpassung des E-Moduls der Sinterpartikel erwartet, ebenfalls im richtigen Verhältnis zu den Kontaktpartnern. Der E-Modul von Sinter wurde in der ursprünglichen Simulation aus simulationstechnischen Gründen möglichst klein gewählt. Aufgrund der Heterogenität des Sinters und der unregelmäßi- 

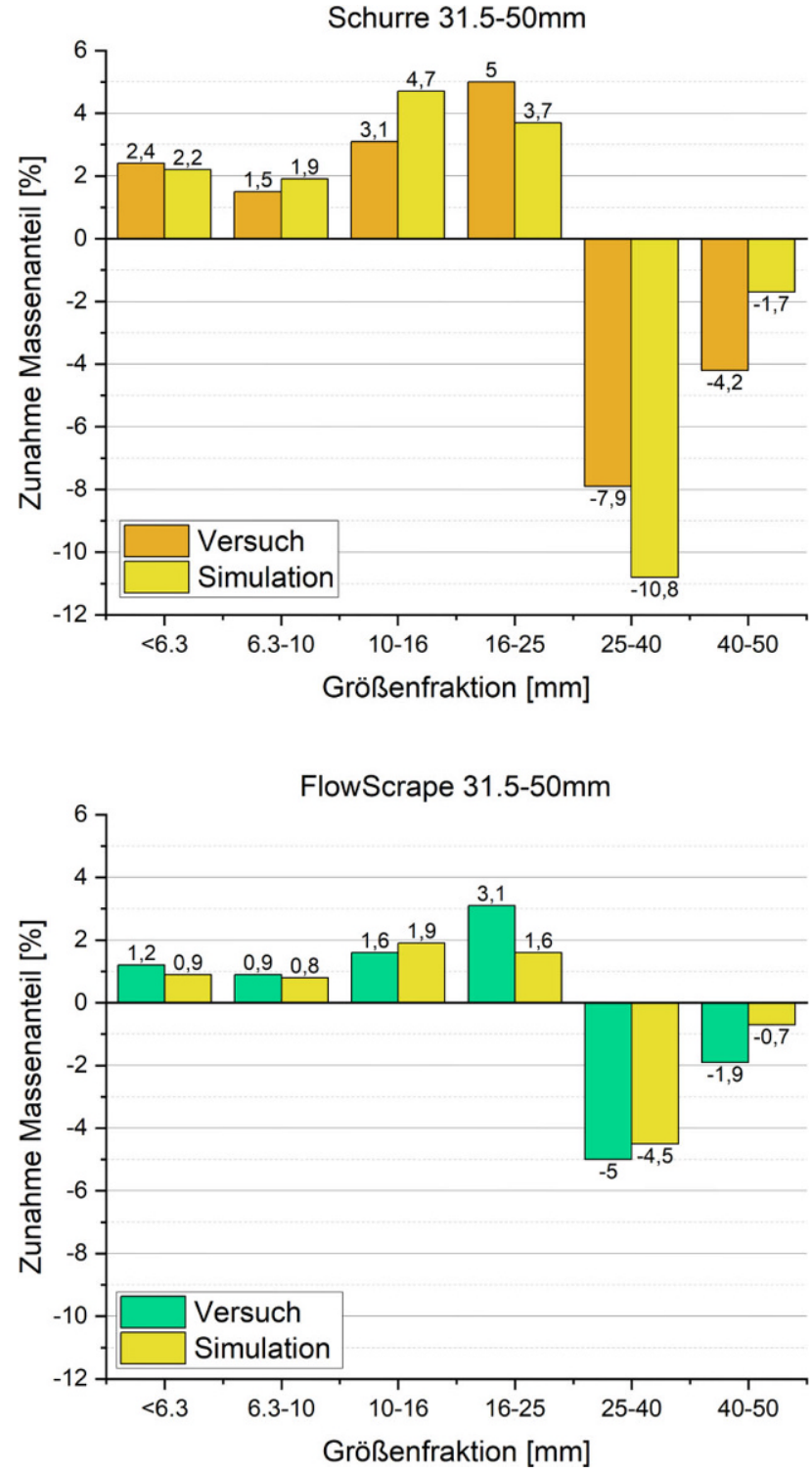

Abb. 14: Partikelbruchvorhersage mittels DEM im Vergleich zum Versuch für die Größenfraktion 31,5-50 mm

gen Form der Sinterpartikel kann der E-Modul nicht exakt ermittelt werden. In [10] wurde versucht durch Auswertung von Druckversuchen, bei denen Kraft und Verformung mitgemessen wurden, einen gemittelten E-Modul für Sinterpartikel zu ermitteln. Die Simulationen mit angepasstem E-Modul ergaben allerdings nur eine geringfügige Verbesserung gegenüber der ursprünglichen Simulation mit kleinerem E-Modul der Partikel. Dies lässt vermuten, dass bei den hier untersuchten Fördervorgängen für den Volumenbruch die Interaktionen zwischen den Partikeln eine untergeordnete Rolle gegenüber den Interaktionen mit den Fördermitteln bzw. den Übergabesystemen spielen. In [1] wird ebenfalls eine Verbesserung der Simulationsergebnisse erwartet, wenn die Partikelgrößenverteilung in der Simulation der tatsächlichen Partikelgrößenverteilung im Schüttgut angepasst wird, anstatt mit einem gemittelten Durchmesser zu simulieren. Dazu müssten allerdings eine feinere
Klassierung der Größenfraktionen erfolgen und die Bruchversuche dementsprechend wiederholt werden.

\section{Ausblick}

Ein Nachteil dieses einfachen Partikelbruchmodells ist, dass ein Partikel nach einem Bruch nicht als gebrochen berücksichtigt werden kann. Dies ist zwar bei einfachen Fördervorgängen mit wenigen Partikeln vernachlässigbar, ist aber weniger geeignet für komplexe Vorgänge mit größeren Massenströmen. Um den Partikelbruch und vor allem die Feinguterzeugung mittels DE-Simulation genauer vorherzusagen, wird derzeit ein neues Partikelbruchmodell entwickelt, welches auf der "Particle Replacement Method" [11-14] kombiniert mit der Voronoi-Tesselation [15-17] basiert. Dieses wird anhand der Ergebnisse aus den Bruchversuchen einfach zu kalibrieren sein. Das neue Bruchmodell ermöglicht es, den Partikelbruch selbst zu simulieren und ebenfalls bruchfähige Fragmente zu generieren, damit auch längere und komplexere Fördervorgänge mit höherer Genauigkeit simuliert werden können.

Danksagung. Diese Arbeit wurde im Rahmen des Projekts MinSiDeg durchgeführt, welches vom Research Fund for Coal and Steel (RFCS) der Europäischen Union unter der Grant Agreement Number 847285 gefördert wurde.

Funding. Open access funding provided by Montanuniversität Leoben.

Open Access Dieser Artikel wird unter der Creative Commons Namens nennung 4.0 International Lizenz veröffentlicht, welche die Nutzung, Vervielfältigung, Bearbeitung, Verbreitung und Wiedergabe in jeglichem Medium und Format erlaubt, sofern Sie den/die ursprünglichen Autor(en) und die Quelle ordnungsgemäß nennen, einen Link zur Creative Commons Lizenz beifügen und angeben, ob Änderungen vorgenommen wurden.

Die in diesem Artikel enthaltenen Bilder und sonstiges Drittmaterial unterliegen ebenfalls der genannten Creative Commons Lizenz, sofern sich aus der Abbildungslegende nichts anderes ergibt. Sofern das betreffende Material nicht unter der genannten Creative Commons Lizenz steht und die betreffende Handlung nicht nach gesetzlichen Vorschriften erlaubt ist, ist für die oben aufgeführten Weiterverwendungen des Materials die Einwilligung des jeweiligen Rechteinhabers einzuholen.

Weitere Details zur Lizenz entnehmen Sie bitte der Lizenzinformation auf http://creativecommons.org/licenses/by/4.0/deed.de.

\section{Literatur}

1. Denzel, M., Prenner, M.: Dynamisches Übergabesystem zur Reduk tion des Partikelbruchs. In: 25. Fachtagung Schüttgutfördertechnik 2021, S. 233-242. (2021) https://doi.org/10.25673/36794

2. Dünnwald, W., Prenner, M.: Vorrichtung zum Leiten eines von einem Abwurfende oder Austragsende einer Fördereinrichtung abfließenden Materialstroms (2019). Patentnr. DE 102019108687 A1. Deutschland

3. Prenner, M.: Dynamische Übergabeschurre zur Effizienzsteigerung von Gurtbandförderern. Berg Huttenm. Monatsh. (2021). https://doi. org/10.1007/s00501-021-01084-4

4. Kogler, S.: Schüttgutübergabeeinrichtung für kritische Randbedin gungen. Montanuniversität Leoben, (2020). Masterarbeit

5. Denzel, M., Prenner, M.: Minimierung des Sinterzerfalls mittels DEM. Berg Huettenm. Monatsh. 166(2), 76-81 (2021). https://doi. org/10.1007/s00501-021-01081-7 
6. Denzel, M., Prenner, M., Sifferlinger, N.A.: Development of an automated single particle impact tester for iron ore sinter. Miner. Eng. 175, 107291 (2022). https://doi.org/10.1016/j.mineng.2021.107291

7. Prenner, M.: Simulationsparameterstudie - Sinterbunker. Montanuniversität Leoben, (2018). Projektbericht

8. Brugger, M.: Rücksprungverhalten von Hochofensinter. Montanuniversität Leoben, (2021). Bachelorarbeit

9. Kogler, P.: Analyse von Kontaktvorgängen und Optimierung von fördertechnischen Anlagen hinsichtlich Partikelbruchs bei Sinter. Montanuniversität Leoben, (2020). Masterarbeit

10. Wagner, P.: Vergleich zweier Übergabeeinrichtungen in Bezug auf Partikelbruch. Montanuniversität Leoben, (2022). Masterarbeit

11. Tavares, L.M., das Chagas, A.S.: A stochastic particle replacement strategy for simulating breakage in DEM. Powder Technol. 377, 222-232 (2021). https://doi.org/10.1016/j.powtec.2020.08.091

12. Cleary, P.: Modelling comminution devices using DEM. Int. J. Numer. Anal. Meth. Geomech. 25(1), 83-105 (2001). https://doi.org/10. 1002/1096-9853(200101)25:1<83::AID-NAG120>3.0.CO;2-K

13. Cleary, P.W.: Recent advances in dem modelling of tumbling mills. Miner. Eng. 14(10), 1295-1319 (2001). https://doi.org/10.1016/S08926875(01)00145-5
14. Jiménez-Herrera, N., Barrios, G.K., Tavares, L.M.: Comparison of breakage models in DEM in simulating impact on particle beds. Adv. Powder Technol. 29(3), 692-706 (2017). https://doi.org/10.1016/j.apt. 2017.12.006

15. Kumar, S., Kurtz, S.K.: Properties of a two-dimensional PoissonVoronoi tesselation: a Monte-Carlo study. Mater. Charact. 31(1), 55-68 (1993). https://doi.org/10.1016/1044-5803

16. Riedinger, R., Habar, M., Oelhafen, P., Güntherodt, H.: About the Delaunay-Voronoi tesselation. J. Comput. Phys. 74(1), 61-72 (1988). https://doi.org/10.1016/0021-9991

17. Kumar, S., Kurtz, S.K.: Simulation of material microstructure using a 3D voronoi tesselation: calculation of effective thermal expansion coefficient of polycrystalline materials. Acta Metall. Mater. 42(12), 3917-3927 (1994). https://doi.org/10.1016/0956-7151(94)90170-8

Hinweis des Verlags. Der Verlag bleibt in Hinblick auf geografische Zuordnungen und Gebietsbezeichnungen in veröffentlichten Karten und Institutsadressen neutral. 